\title{
Magnetic Properties of Mechanochemically Synthesized Mixed Oxides
}

\begin{abstract}
N.G. Kostova ${ }^{a}$, A. Zorkovská ${ }^{b, *}$, J. KováČ ${ }^{b}$, N. Velinov ${ }^{a}$, P. Baláž $\check{Z}^{b}$
${ }^{a}$ Institute of Catalysis, Bulgarian Academy of Sciences, acad. G. Bonchev, Bl. 11, 11113 Sofia, Bulgaria

${ }^{b}$ Institute of Geotechnics Slovak Academy of Sciences, Watsonova 45, 04353 Košice, Slovakia

The mixed oxides $\mathrm{Fe}_{2} \mathrm{O}_{3}-\mathrm{ZnO}$ have been obtained in nanocrystalline state by reactive milling in a high-energy planetary mill, from a stoichiometric mixture of hematite and $\mathrm{ZnO}$. The magnetic properties of samples were evaluated by magnetization measurements and Mössbauer spectrometry. A post milling annealing promotes the solid state reaction and improves the zinc ferrite formation, paramagnetic $\mathrm{ZnFe}_{2} \mathrm{O}_{4}$ phase is formed. Further mechanical activation leads to structural transformation into Wüstite-type (FeZn)O mixed oxide, with ferromagnetic hysteresis and increased magnetization.
\end{abstract}

DOI: 10.12693/APhysPolA.126.411

PACS: 77.84.Bw, 75.60.-d, 76.80.+y, 61.05.cp, 81.07.Wx

\section{Introduction}

Mixed oxides are the oxygen-containing combination of two or more metallic ions. They have many industrial applications in electronics, ceramics and catalysis. Wide-gap diluted magnetic oxide semiconductors, like the $\mathrm{Fe}_{2} \mathrm{O}_{3}-\mathrm{ZnO}$ mixed oxide, combine their electric conductivity with ferromagnetism and optical transparency, thereby opening up the possibility of new device concepts. Spinel ferrites have very important technological applications in sensor techniques, medical diagnostics, drug-delivery and information storage. Various synthesis methods, such as a chemical route [1] sol-gel combustion method [2], thermal decomposition of metal-surfactant complexes [3] have been used for preparation, which can lead to magnetic properties on diverse scales. Mechanical activation, especially ball milling is a simple and suitable alternative method for preparation of mixed oxides [4]. The aim of this work is to prepare mechanically synthesized $\mathrm{Fe}_{2} \mathrm{O}_{3}-\mathrm{ZnO}$ mixed oxides and characterize their magnetic properties.

\section{Experimental}

Mechanochemical synthesis of $\mathrm{Fe}_{2} \mathrm{O}_{3}-\mathrm{ZnO}$ mixed oxides was performed in a laboratory planetary mill Pulverisette 6 (Fritsch, Germany) by high-energy milling of hematite and $\mathrm{ZnO}$ in stoichiometric ratio 1:1. For the mechanochemical synthesis the following experimental conditions were applied: loading of the mill, 50 balls of $10 \mathrm{~mm}$ in diameter; material of milling chamber and balls was tungsten carbide; volume of milling chamber, $250 \mathrm{ml}$; room temperature; rotational speed of the mill planet carrier $400 \mathrm{~min}^{-1}$; milling time, $10 \mathrm{~min}$. dry milling and subsequent wet milling in the presence of ethylcellulose. Sample 1 is as-synthesized, Sample 2 is annealed at $600^{\circ} \mathrm{C}$, Sample 3 is annealed at $700^{\circ} \mathrm{C}$, and additionally mechanically activated for $4 \mathrm{~h}$.

X-ray powder diffraction patterns (XRD) of the samples were taken at room temperature using a D8 Advance

\footnotetext{
*corresponding author; e-mail: zorkovska@saske.sk
}

diffractometer (Bruker, Germany), equipped with a $\Theta / \Theta$ goniometer, $\mathrm{Cu}-\mathrm{K}_{\alpha}$ radiation, secondary graphite monochro-mator and scintillation detector in BraggBrentano geometry. The diffraction data were collected over an angular range $15^{\circ}<2 \Theta<80^{\circ}$ with steps of $0.03^{\circ}$ and a fixed counting time of $25 \mathrm{~s} / \mathrm{step}$. For the phase identification and full profile fitting the Diffrac ${ }^{p l u s}$ Eva and Diffrac ${ }^{\text {plus }}$ Topas software has been utilized, respectively.

Transmission ${ }^{57} \mathrm{Fe}$ Mössbauer spectra were obtained at room temperature with a Wissel electromechanical Mössbauer spectrometer (Wissenschaftliche Electronik $\mathrm{GmbH}$, Germany) working at a constant acceleration mode. $\mathrm{A}{ }^{57} \mathrm{Co} / \mathrm{Cr}$ source and a $\alpha$-Fe foil standard were used.

The magnetization curves were investigated using a vibrating sample magnetometer, operating in magnetic fields up to $6 \mathrm{~T}$ in temperature range of 4-290 K.

\section{Results and discussion}

XRD analysis (Fig. 1) reveals the different phase composition of the samples. Mechanical activation at the given conditions does not lead to complete conversion of reagents to mixed oxide. The product consists of un-

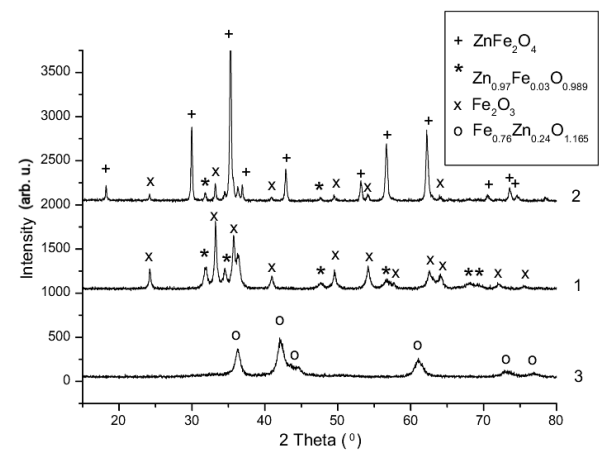

Fig. 1. X-ray diffraction spectra of the samples: (1) as-mechano-synthesized (2) annealed at $600^{\circ} \mathrm{C}(3)$ annealed at $700{ }^{\circ} \mathrm{C}$ and additionally mechanically activated. 


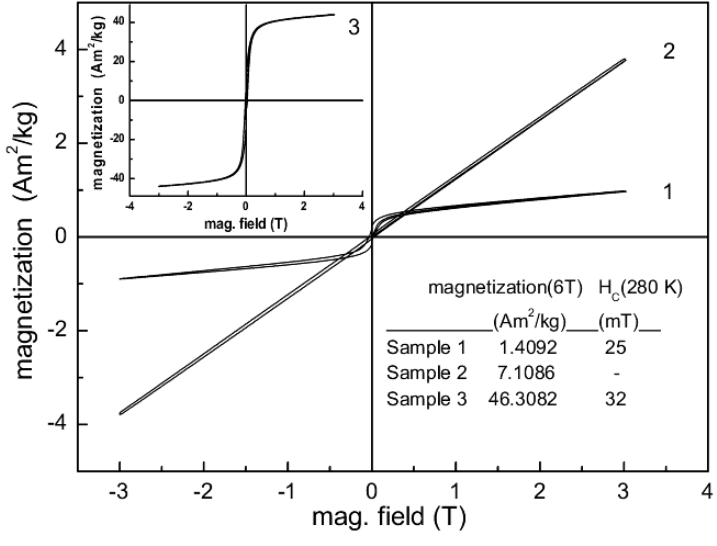

Fig. 2. Room temperature magnetization curves of the samples.

reacted hematite and $\mathrm{ZnO}$, with a small degree of $\mathrm{Fe}$ incorporation. The average crystallite size is between 10-20 nm. Annealing of the product at $600{ }^{\circ} \mathrm{C}$ leads to the formation of ferrite type mixed oxide $\mathrm{ZnFe}_{2} \mathrm{O}_{4}$ with crystallite size of about $60 \mathrm{~nm}$, but considerable amount of reagents remains unreacted. Annealing at $700{ }^{\circ} \mathrm{C}$ promotes the formation of zinc ferrite, which is the major phase (not shown). Further mechanical activation re- sulted in Wüstite-type tetragonally distorted mixed oxide, with prevalence of $\mathrm{Fe}$, and fine crystalline structure in the range below $10 \mathrm{~nm}$.

Mössbauer technique was used to study the magnetic properties and ionic state of the iron in $\mathrm{Fe}_{2} \mathrm{O}_{3}-\mathrm{ZnO}$ mixed oxides (Table). The measured spectra represent doublets or combination of a doublet $(\mathrm{Db})$ and sextet $(\mathrm{Sx})$. The hyperfine parameters for the sextets are in correspondence with those for $\mathrm{Fe}^{3+}$ ions, octahedrally coordinated in hematite $\left(\alpha-\mathrm{Fe}_{2} \mathrm{O}_{3}\right.$-substance with antiferromagnetic or weakly ferromagnetic properties). The parameters of the doublet components are those of the $\mathrm{Fe}^{3+}$ ions of octahedral coordination in the paramagnetic $\mathrm{ZnFe}_{2} \mathrm{O}_{4}$ phase. One can estimate the approximate ratio between the iron-containing phases in the samples based on the ratio between the relative weights of the components $G$ (Table).

The results of magnetization measurements at room temperature are summarized in Fig. 2. Sample 1 shows hysteresis (coercivity $H_{C} \sim 25 \mathrm{mT}$ ) and its magnetization does not saturate in magnetic field of 6 T. Sample 2 is paramagnetic at room temperature, however, at $4 \mathrm{~K}$, hysteresis loops with $H_{C} \sim 80 \mathrm{mT}$ have been observed (not shown) and the magnetization does not saturate as well. Sample 3 has the largest magnetization and shows ferromagnetic-like hysteresis with $H_{C} \sim 32 \mathrm{mT}$.

TABLE

Parameters of the Mössbauer spectra of the investigated samples. IS-isomer shift, QS-quadrupole splitting, $H_{e f f}$-hyperfine field, FWHM-full width at half maximum, $G$-relative weights.

\begin{tabular}{c|c|c|c|c|c|c}
\hline \hline Sample code & Components & $\begin{array}{c}\text { IS } \\
(\mathrm{mm} / \mathrm{s})\end{array}$ & $\begin{array}{c}\text { QS } \\
(\mathrm{mm} / \mathrm{s})\end{array}$ & $\begin{array}{c}H_{\text {eff }} \\
(\mathrm{T})\end{array}$ & $\begin{array}{c}\text { FWHM } \\
(\mathrm{mm} / \mathrm{s})\end{array}$ & $\begin{array}{c}G \\
(\%)\end{array}$ \\
\hline Sample 1 & $\mathrm{Sx}-\alpha-\mathrm{Fe}_{2} \mathrm{O}_{3}-\mathrm{Fe}^{3+}$ & 0.37 & -0.17 & 51.5 & 0.37 & 98 \\
& $\mathrm{Db}-\mathrm{ZnFe}_{2} \mathrm{O}_{4}-\mathrm{Fe}^{3+}$ & 0.35 & 0.40 & - & 0.46 & 2 \\
\hline Sample 2 & $\mathrm{Sx} \alpha-\mathrm{Fe}_{2} \mathrm{O}_{3}-\mathrm{Fe}^{3+}$ & 0.38 & -0.19 & 51.6 & 0.28 & 22 \\
& $\mathrm{Db}-\mathrm{ZnFe}_{2} \mathrm{O}_{4}-\mathrm{Fe}^{3+}$ & 0.35 & 0.39 & - & 0.36 & 78 \\
\hline Sample 3 before & $\mathrm{Db}-\mathrm{ZnFe}_{2} \mathrm{O}_{4}-\mathrm{Fe}^{3+}$ & 0.35 & 0.40 & - & 0.41 & 100 \\
second milling & & & & & & \\
\hline
\end{tabular}

\section{Conclusions}

Mechanochemical reaction between hematite and $\mathrm{ZnO}$ in planetary mill formed a precursor, which was subsequently heated to successfully produce $\mathrm{ZnFe}_{2} \mathrm{O}_{4}$. The reaction begins after the amorphization of $\mathrm{ZnO}$, whereas $\alpha-\mathrm{Fe}_{2} \mathrm{O}_{3}$ is still crystalline. It has been found that the obtained milled powder after annealing is a spinel-type structure with paramagnetic behaviour. Additional mechanical activation leads to the formation of Wüstitetype $(\mathrm{FeZn}) \mathrm{O}$ mixed oxide showing ferromagnetic hysteresis and increased magnetization.

\section{Acknowledgments}

This work was supported by the National Science Fund of Bulgaria (project DNTS/Slovakia 01/3), by the Slovak Agency for Science and Development (APVV-0189-10), Ministry of Education of the Slovak Republic (VEGA -
1/0861/12), through a bilateral project between Bulgarian and Slovak Academy of Sciences, and through the European Regional Development Fund (nanoCEXmatI26220120019 and nanoCEXmatII-26220120035).

\section{References}

[1] A.K. Mishra, D. Das, Mater. Sci. Eng. B 171, 5 (2010).

[2] T. Tangcharoen, A. Ruangphanit, W. Pecharapa, Ceramics Inter. 39, S239 (2013).

[3] Ch-Yao, Q. Zheng, G.F. Goya, T. Torres, J. Liu, H.Wu, M. Ge, Y. Zeng, Y. Wang, J.Z. Jiang, J. Phys. Chem. C 111, 12274 (2007).

[4] P. Baláž, Mechanochemistry in Nanoscience and Mineral Engineering, ISBN: 3540748547, SpringerVerlag, Berlin Heidelberg, (2008). 